تأثير كلوريد الصوديوم ودرجة الحرارة على بكتريا Brucella melitensis المعزولة من دم الانسان وحليب الاغنام

$$
\text { قسم علوم الاغذية والتيقانات الاحيائية / كلية الزراعة والغابات / جامعة الموصل / ابر / العراق اقل }
$$

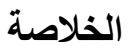

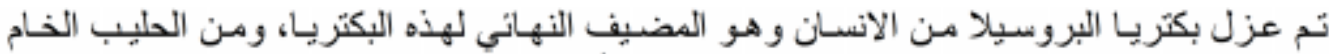

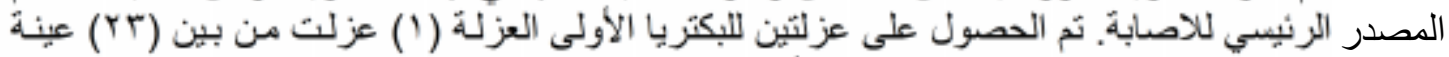

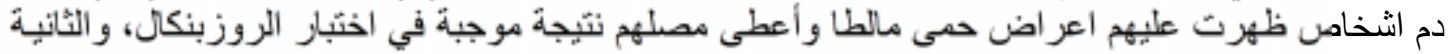

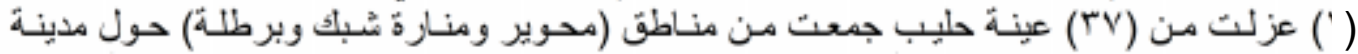

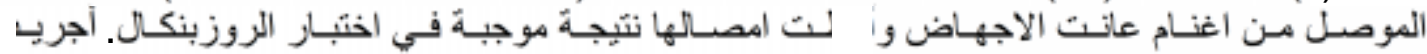

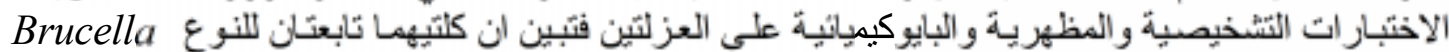
melitensis

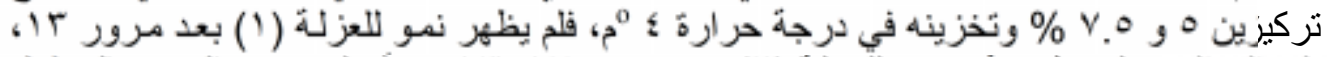

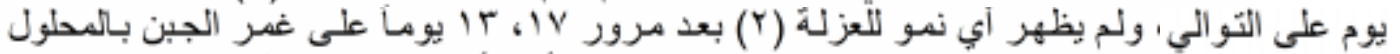

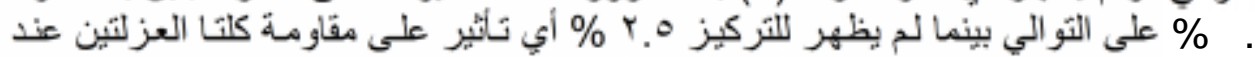

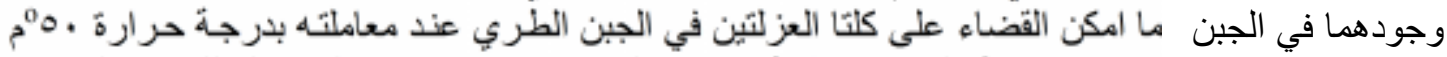

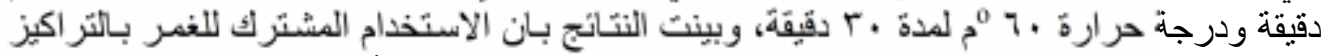

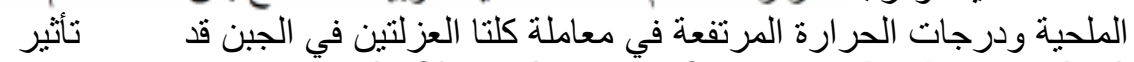

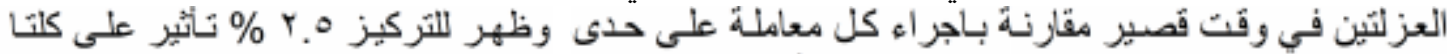

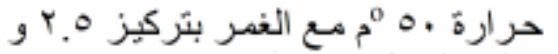

$$
\text { دقائق على التّو التي }
$$

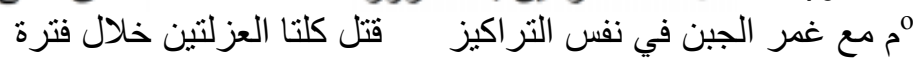

\section{المقدمة - 2 - 20e}

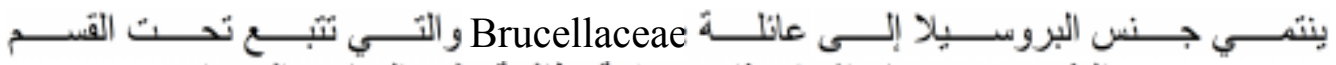
Gandara) Proteobacteria جميعها ممرضة

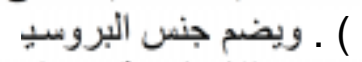
Madigan

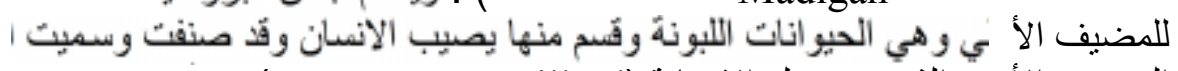

هrucella . (

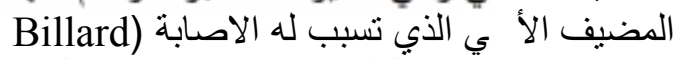

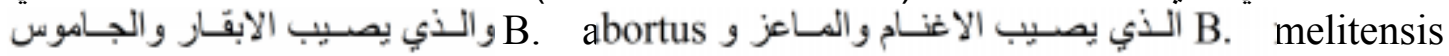

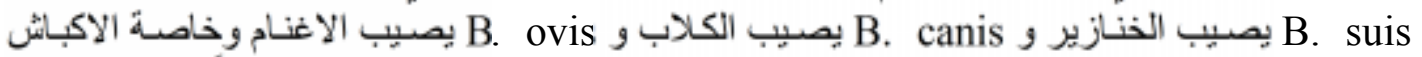
B. maris

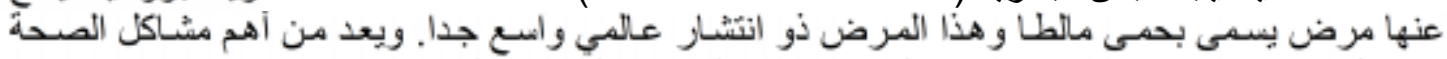

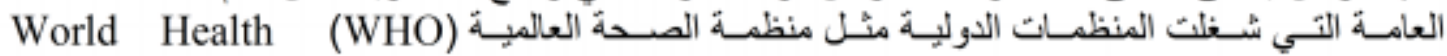

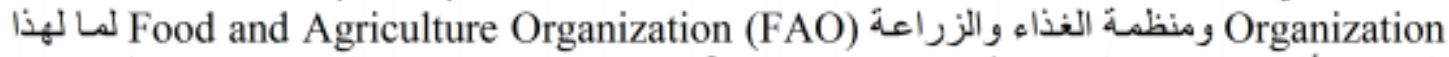

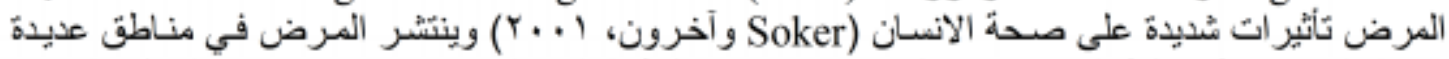

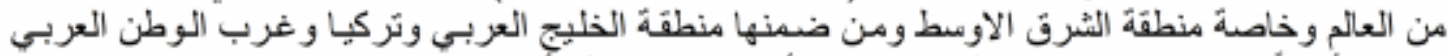

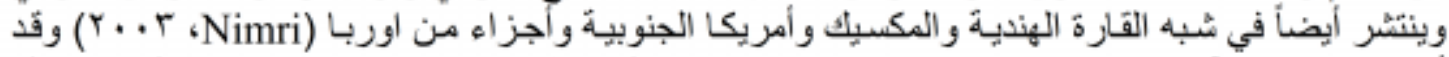

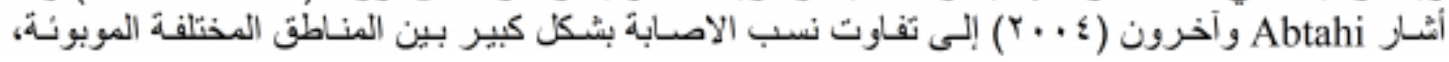




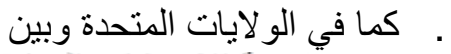

فهي تتر اوح ما بين نسبة

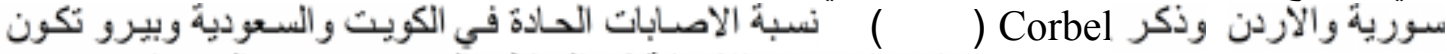

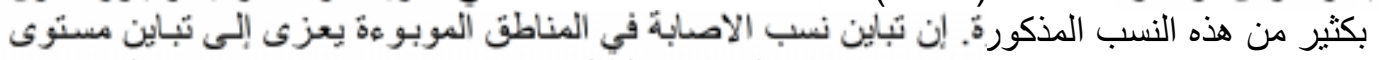

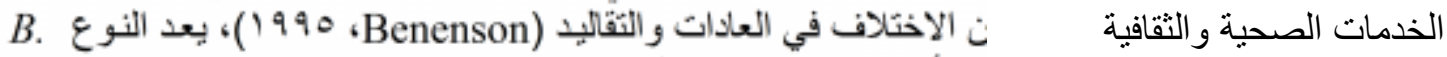

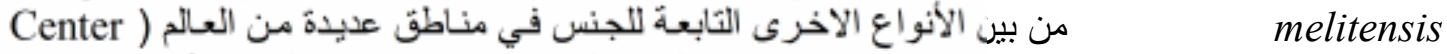

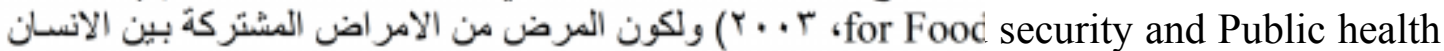

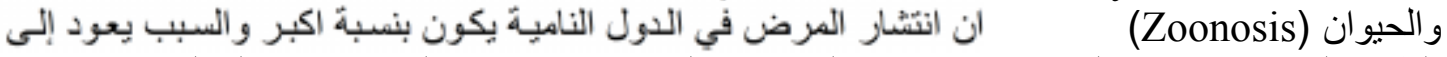

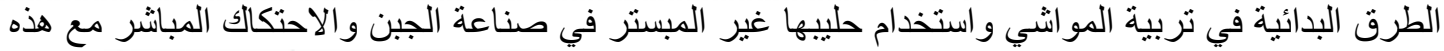

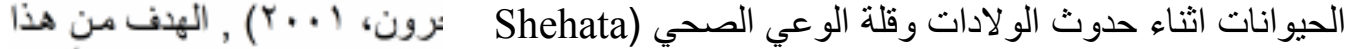

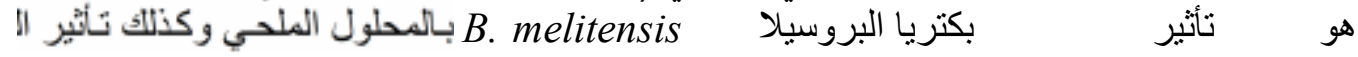
بدرجات الحر ارية و المعاملة بكلا العاملين معا.

المواد البحث وطرائقه

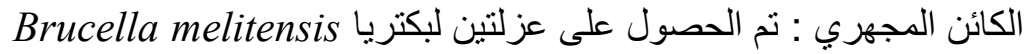

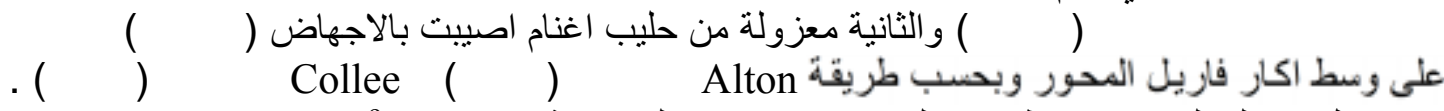

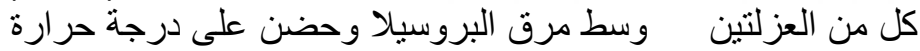

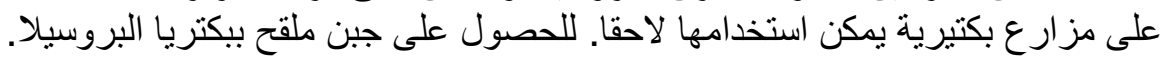

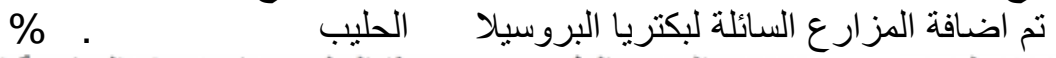

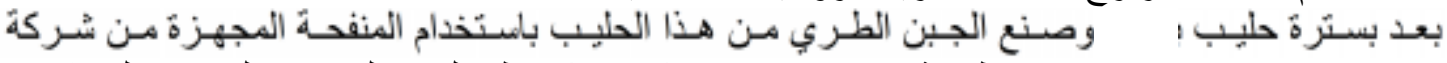

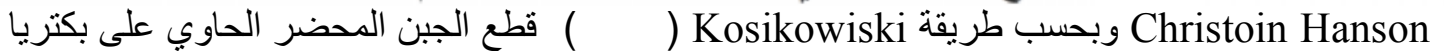

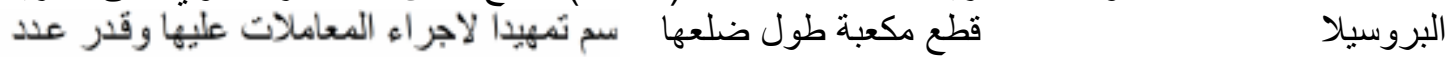

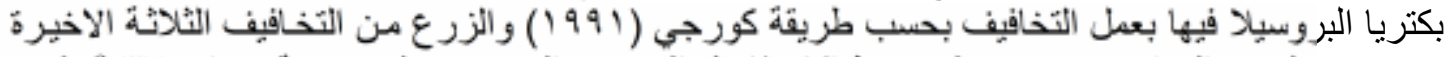

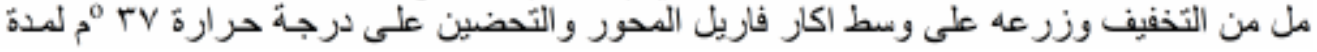

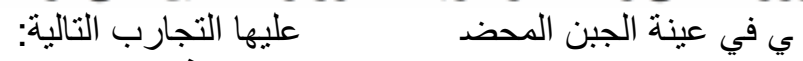

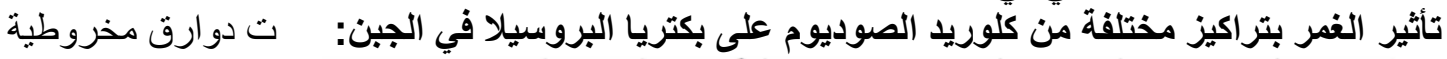

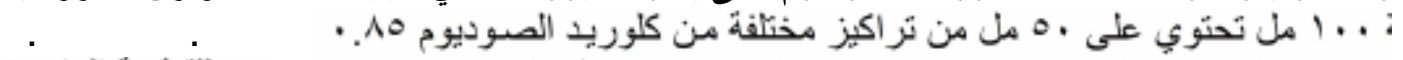

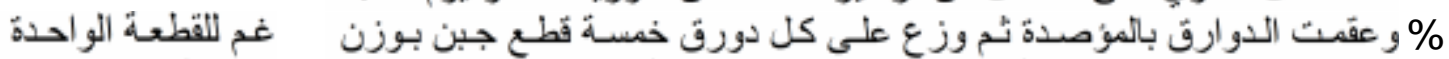

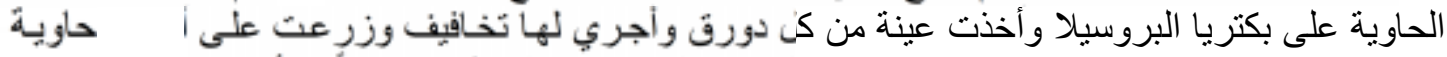

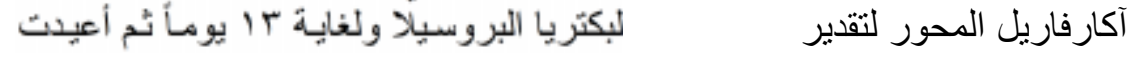

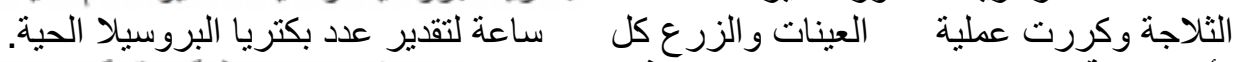

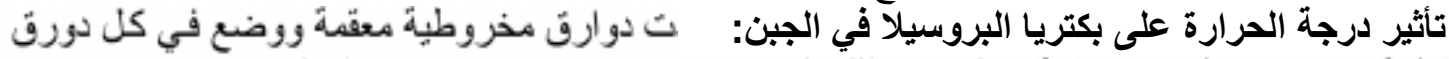

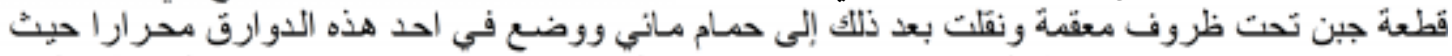

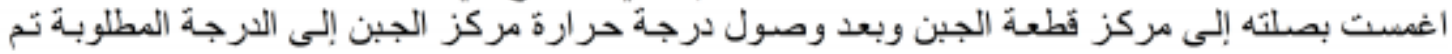

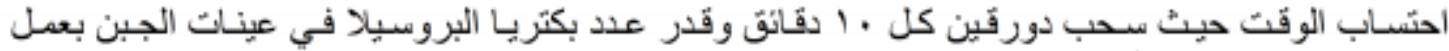

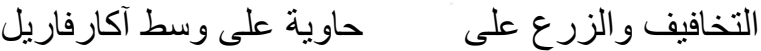

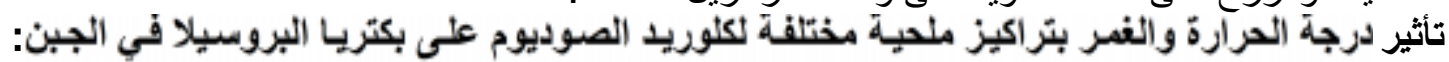

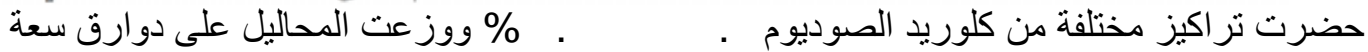

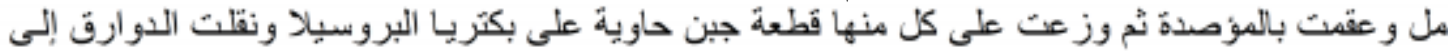

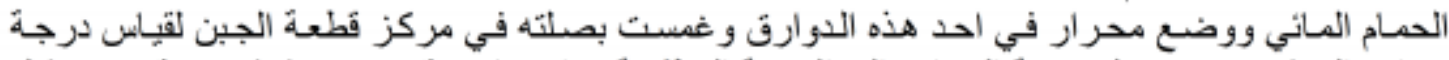

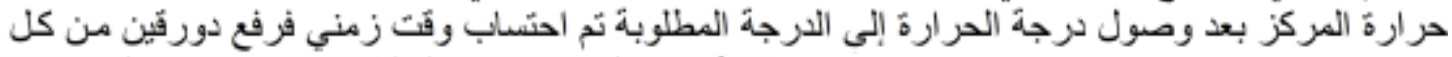

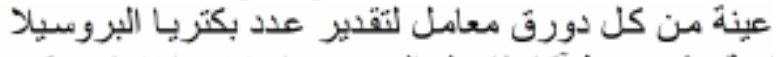
دقيقة. بعدها تركيز كل

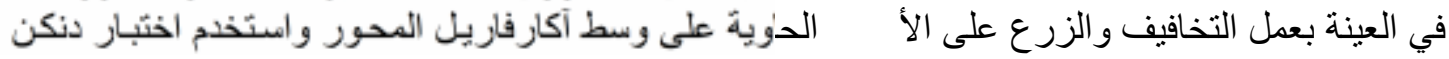



( ) ( )

تأثير الغمر بتراكيز مختلفة من كلوريد الصوديوم على بكتريا البروسيلا في الجبن: يوضح الجدولين

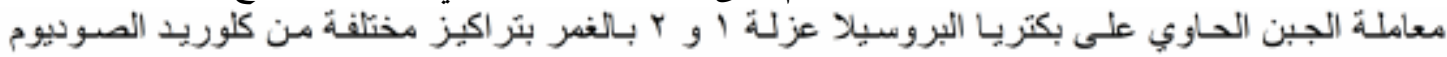

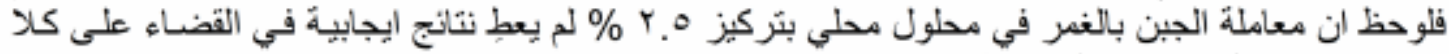

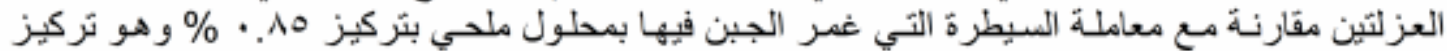

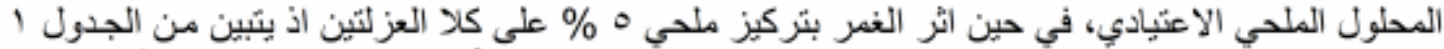

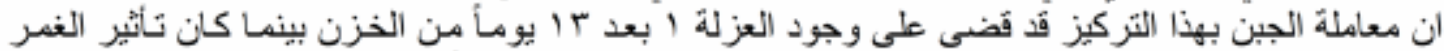

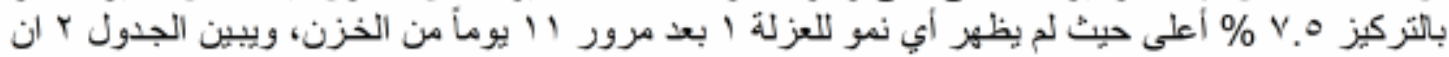

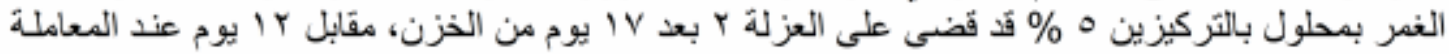

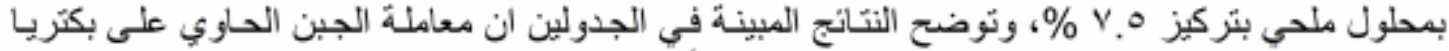

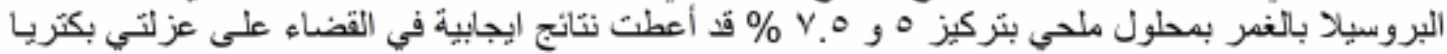

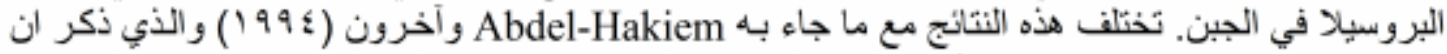

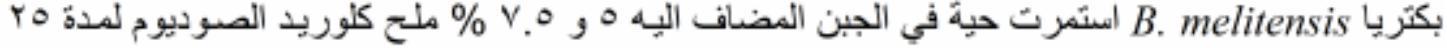

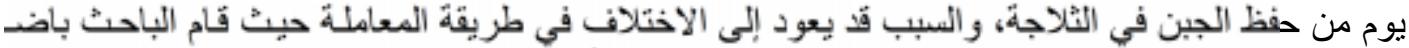

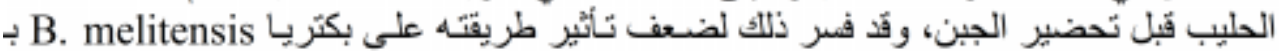

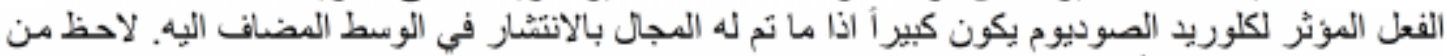

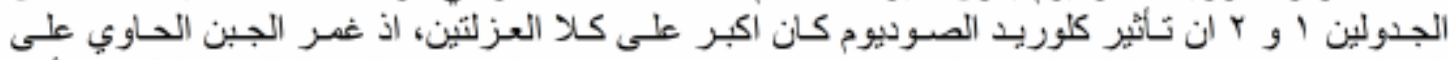

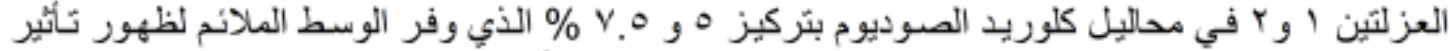

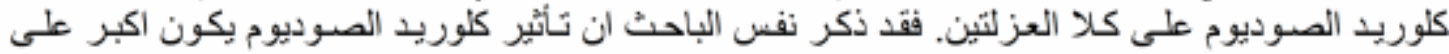
الاحياء المجهرية المرضية عند اذابته بالماء المقطر مقارنة مع اذابته في وسط كالحليب.

( ) : تأثير الغمر بتر اكيز مختلفة من كلوريد الصوديوم على بكثريا البروسيلا العزلة ( ) )

\begin{tabular}{|c|c|c|c|c|}
\hline & & & \multicolumn{2}{|c|}{ /CUF } \\
\hline \multicolumn{4}{|c|}{ كلوريد الصوديوم (\%) } & \multirow[b]{2}{*}{ (يوم) } \\
\hline . & & . & . & \\
\hline$x$ & $x$ & $x$ & $x$ & \\
\hline$x$ & $x$ & $x$ & $x$ & \\
\hline$x$ & $x$ & $x$ & $x$ & \\
\hline$x$ & $x$ & $x$ & $x$ & \\
\hline$x$ & $x$ & $x$ & $x$ & \\
\hline$x$ & $x$ & $x$ & $x$ & \\
\hline$x$ & $x$ & $x$ & $x$ & \\
\hline$x$ & $x$ & $x$ & $x$ & \\
\hline$x$ & $x$ & $x$ & $x$ & \\
\hline$x$ & $x$ & $x$ & $x$ & \\
\hline$x$ & $x$ & $x$ & $x$ & \\
\hline N.g & $x$ & $x$ & $x$ & \\
\hline N.g & $x$ & $x$ & $x$ & \\
\hline N.g & N.g & $x$ & $x$ & \\
\hline
\end{tabular}



()$\quad()$
(ISSN $1815-316 \mathrm{X})$
مجلة زر اعة الر افدين

( ) : تأثثر الغمر بتر اكيز مختلفة من كلوريد الصوديوم على بكتريا البروسيلا العزلة ( ) في الجين

\begin{tabular}{|c|c|c|c|c|}
\hline \multicolumn{4}{|c|}{ وربد الصوديوم (\%) } & \multirow{2}{*}{ 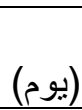 } \\
\hline . & & . & . & \\
\hline$x$ & $x$ & $x$ & $x$ & \\
\hline$x$ & $x$ & $x$ & $x$ & \\
\hline$x$ & $x$ & $x$ & $x$ & \\
\hline$x$ & $x$ & $x$ & $x$ & \\
\hline$x$ & $x$ & $x$ & $x$ & \\
\hline$x$ & $x$ & $x$ & $x$ & \\
\hline$x$ & $x$ & $x$ & $x$ & \\
\hline$x$ & $x$ & $x$ & $x$ & \\
\hline$x$ & $x$ & $x$ & $x$ & \\
\hline$x$ & $x$ & $x$ & $x$ & \\
\hline$x$ & $x$ & $x$ & $x$ & \\
\hline$x$ & $x$ & $x$ & $x$ & \\
\hline$x$ & $x$ & $x$ & $x$ & \\
\hline${ }^{*} \mathrm{~N} . \mathrm{g}$ & $x$ & $x$ & $x$ & \\
\hline N.g & $x$ & $x$ & $x$ & \\
\hline N.g & $x$ & $x$ & $x$ & \\
\hline N.g & $x$ & $x$ & $x$ & \\
\hline N.g & N.g & $x$ & $x$ & \\
\hline
\end{tabular}

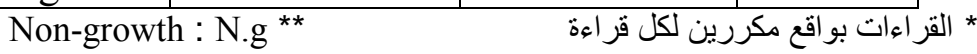

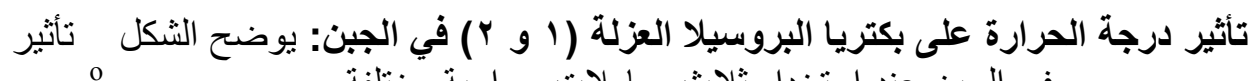

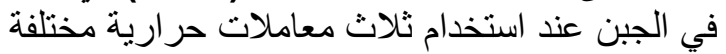

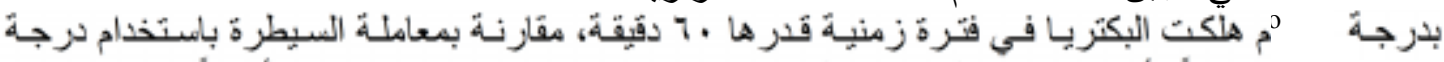

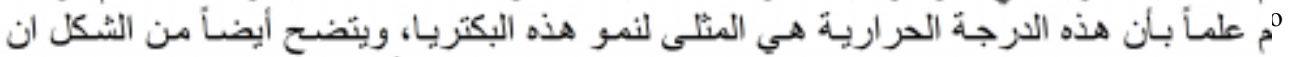

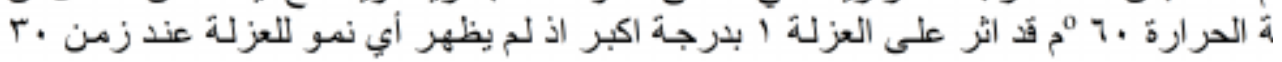

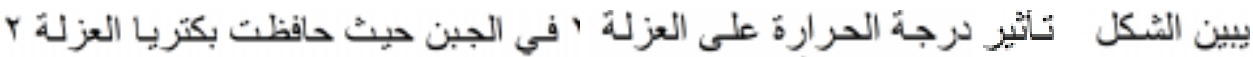

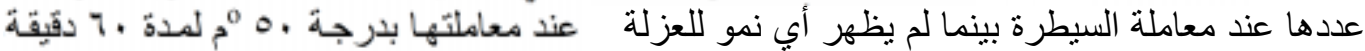

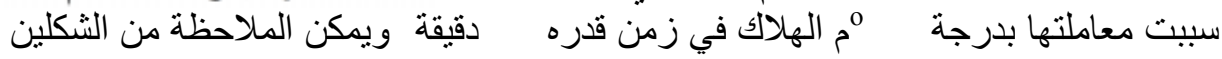

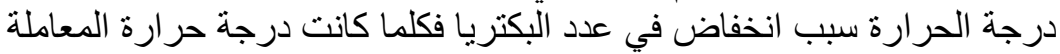
كلتا العزلتين 

( ) ( )

وبين John

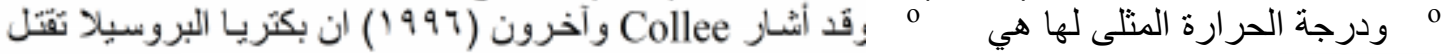

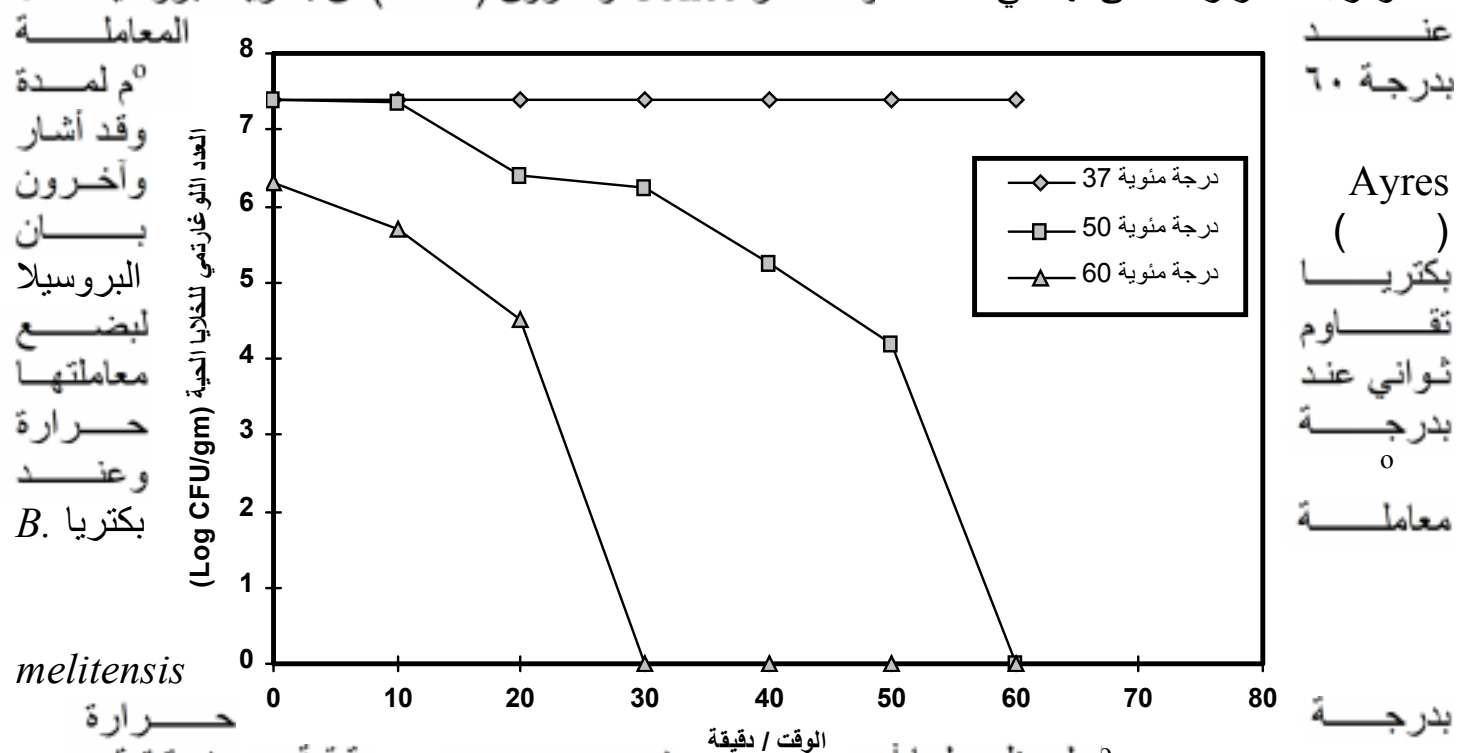

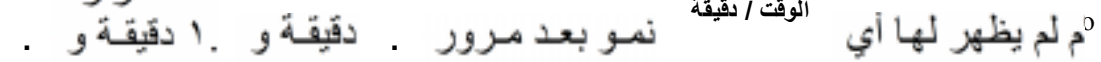

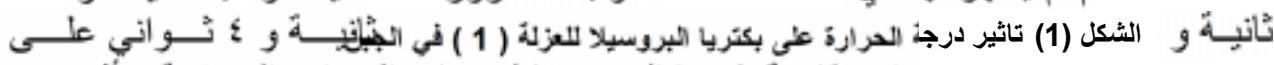

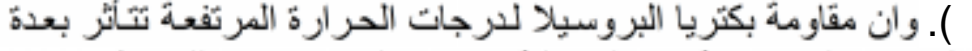

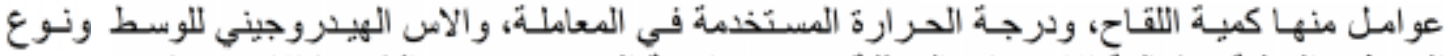

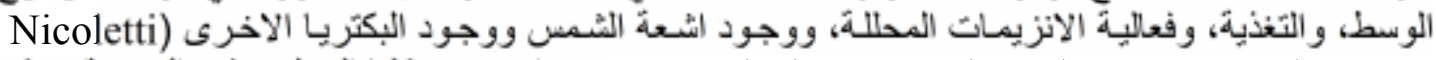

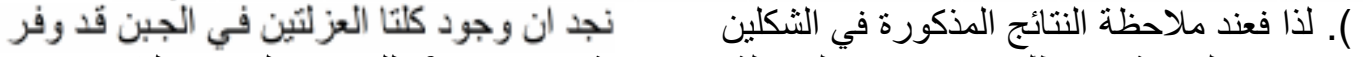

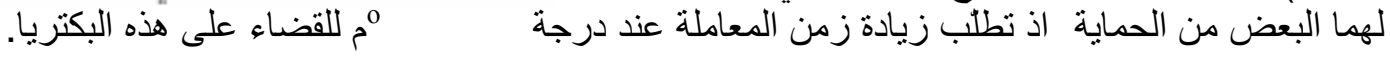

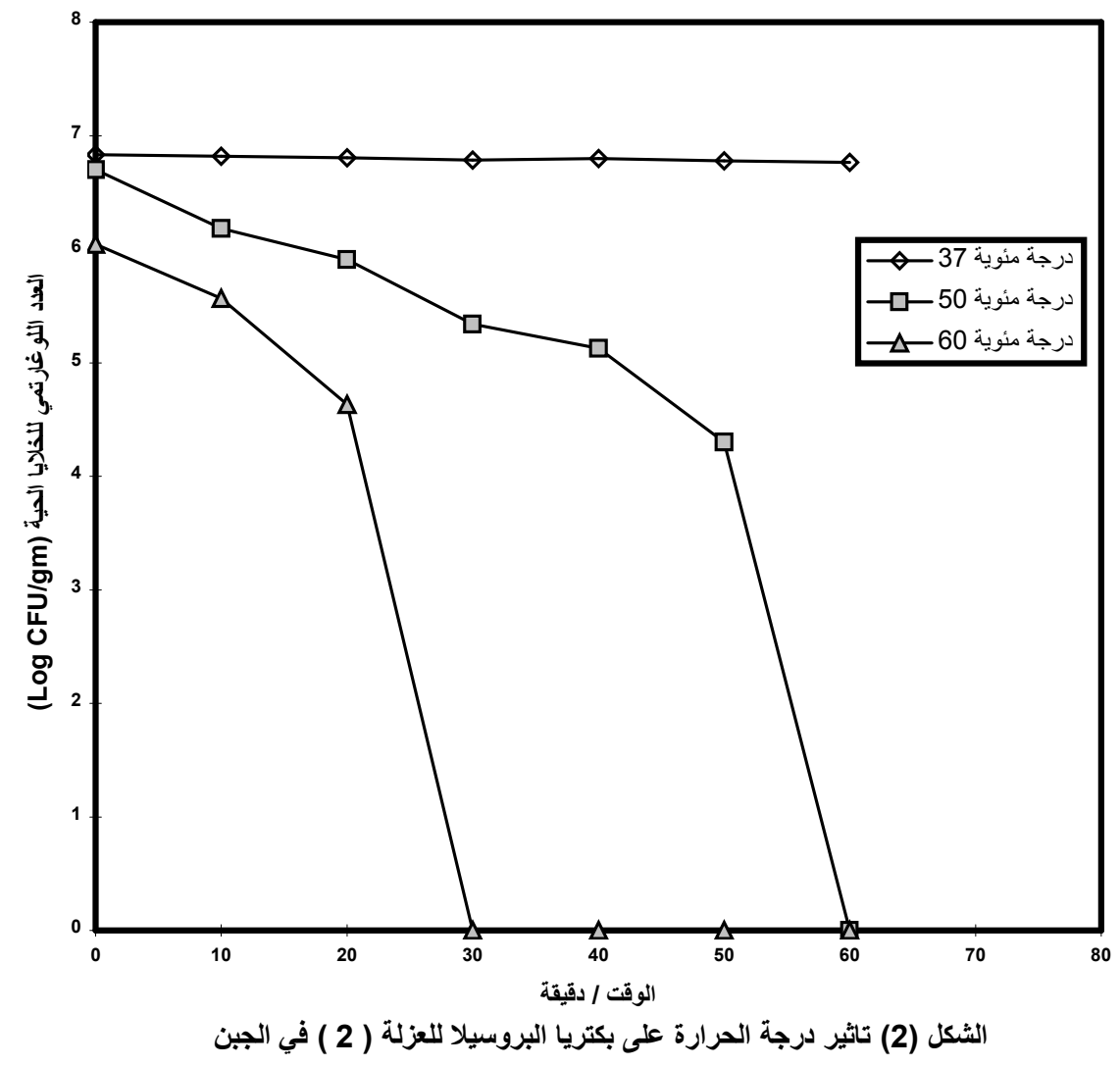




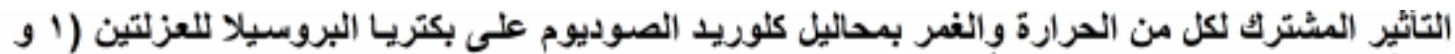

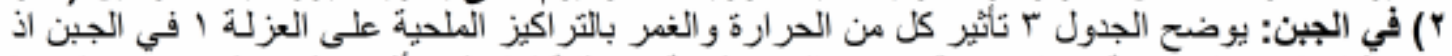

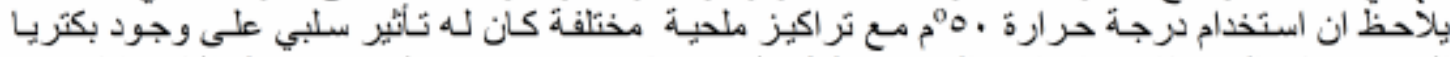

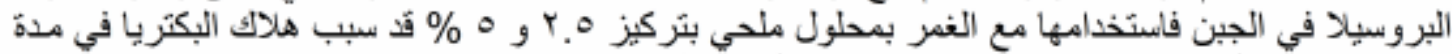

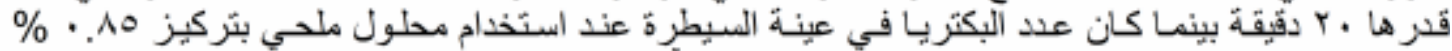

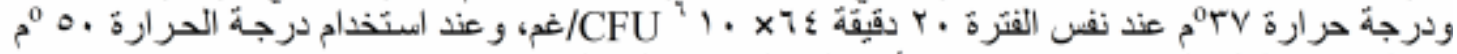

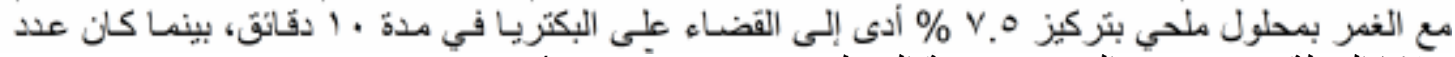

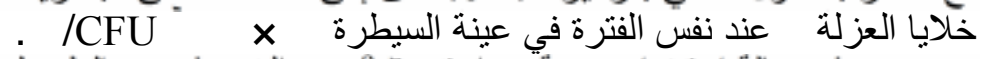

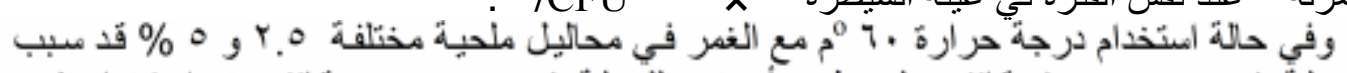

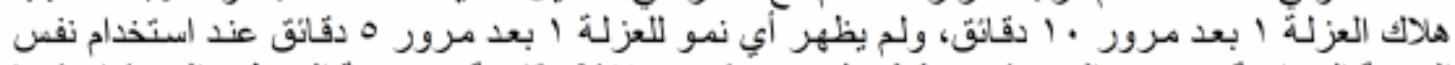

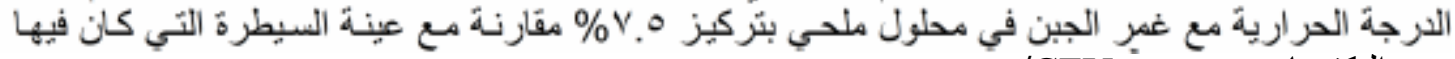

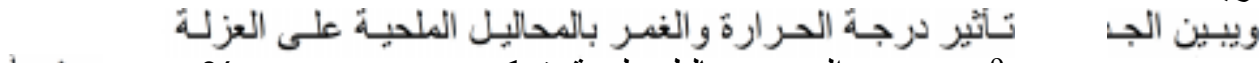

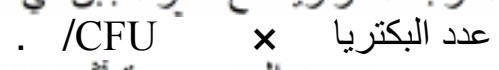

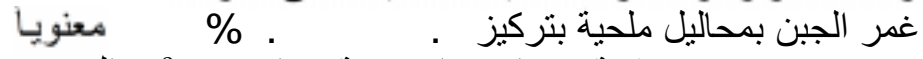

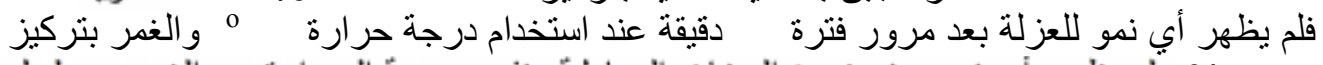

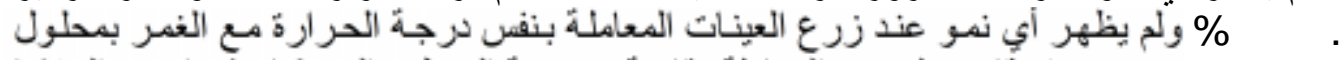

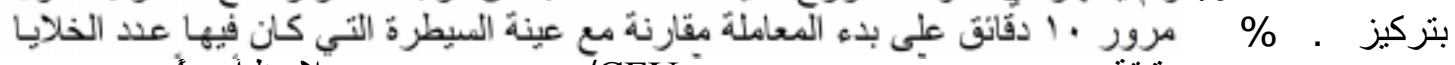
دقيقة

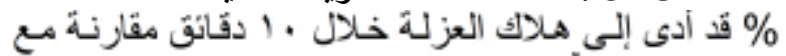

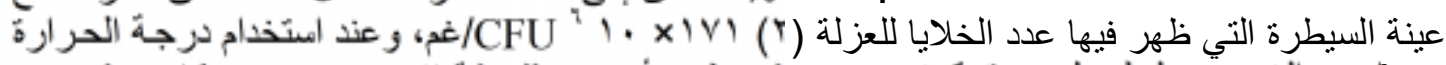

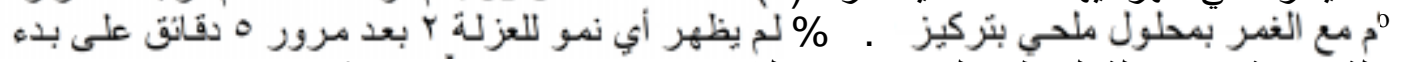

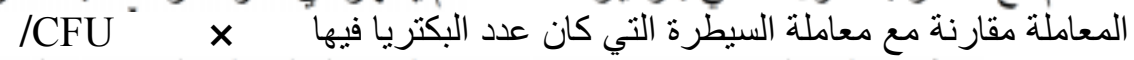

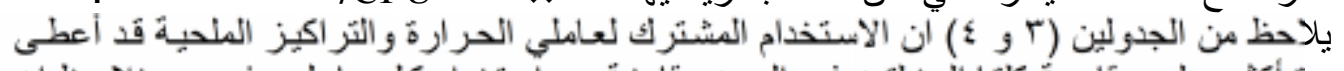

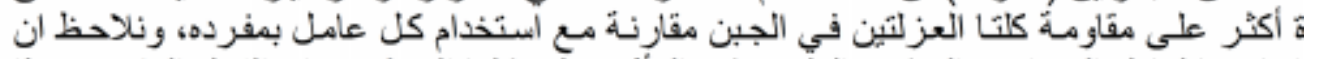

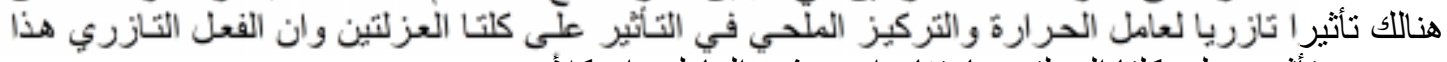

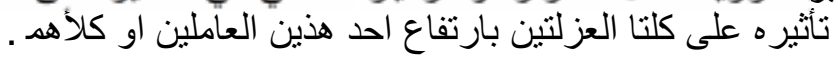

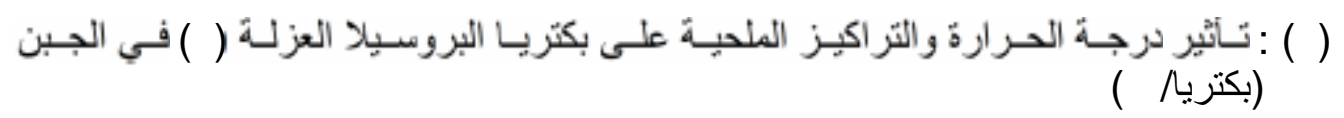



( ) ( )
(ISSN $1815-316 \mathrm{X})$
مجلة زر اعة الر افدين

\begin{tabular}{|c|c|c|c|c|c|c|c|}
\hline \multirow{3}{*}{ م وبتركيز } & \multicolumn{6}{|c|}{ تركيز كلوريد الصوديوم (\%) } & \multirow{3}{*}{ 哥 } \\
\hline & \multicolumn{3}{|c|}{ o } & \multicolumn{3}{|c|}{ o } & \\
\hline & & & 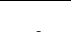 & 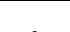 & & . & \\
\hline$x$ & $x$ & $x$ & $x$ & $\times$ & $x$ & * $\quad \times$ & \\
\hline ج د هـ & $N \cdot g^{* *}$ & $x$ & $x$ & $x$ & $x$ & $x$ & \\
\hline$x$ & N.g & N.g & N.g & N.g & $x$ & $x$ & \\
\hline د هـ و & N.g & N.g & N.g & N.g & $x$ & $x$ & \\
\hline$x$ & N.g & N.g & N.g & N.g & N.g & N.g & \\
\hline ج دده & N.g & N.g & N.g & N.g & N.g & N.g & \\
\hline$x$ & N.g & N.g & N.g & N.g & N.g & N.g & \\
\hline هـ و & N.g & N.g & N.g & N.g & N.g & N.g & \\
\hline$x$ & N.g & N.g & N.g & N.g & N.g & N.g & \\
\hline جد هـ & N.g & N.g & N.g & N.g & N.g & N.g & \\
\hline جدهـ & N.g & N.g & N.g & N.g & N.g & N.g & \\
\hline$x$ & N.g & N.g & N.g & N.g & N.g & N.g & \\
\hline$x$ & N.g & N.g & N.g & N.g & N.g & N.g & \\
\hline
\end{tabular}

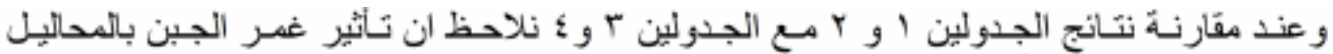

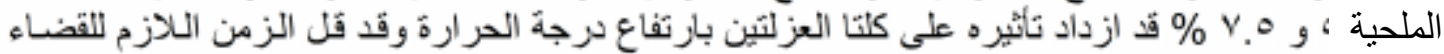

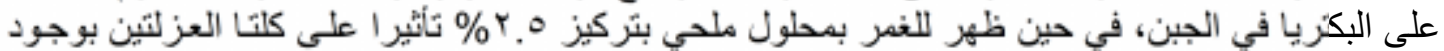
عامل الحرارة بعد ان كان تأثثر هذا التركيز معدوما على كلتا العزلتين عند استخدامه بمفرده.

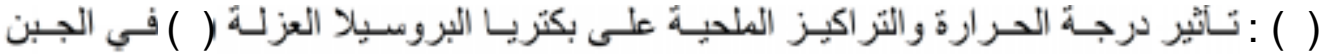

\begin{tabular}{|c|c|c|c|c|c|c|c|c|}
\hline \multicolumn{9}{|c|}{ (بكتريا/ (ب) } \\
\hline \multirow{3}{*}{$\begin{array}{l}\text { o } \\
\end{array}$} & \multirow{3}{*}{ وبتركيز } & \multicolumn{6}{|c|}{ تركيز كلوريد الصوديوم (\%) } & \multirow{3}{*}{ 高 } \\
\hline & & & & \\
\hline & & . & & . & . & & . & \\
\hline هـو ز & $x$ & $x$ & $x$ & $x$ & $x$ & $x$ & * $\quad x$ & \\
\hline & $x$ & $\mathrm{~N} . \mathrm{g}^{\star \star}$ & $\times$ & $\times$ & $\times$ & $x$ & $x$ & \\
\hline & $x$ & N.g & N.g & N.g & N.g & $\times$ & $x$ & \\
\hline د هـ & $x$ & N.g & N.g & N.g & N.g & $x$ & $x$ & \\
\hline جد هـ & $x$ & N.g & N.g & N.g & N.g & N.g & N.g & \\
\hline & $x$ & N.g & N.g & N.g & N.g & N.g & N.g & \\
\hline & $x$ & N.g & $\mathrm{N.g}$ & N.g & N.g & N.g & N.g & \\
\hline ج د هـ & $x$ & N.g & N.g & N.g & N.g & N.g & N.g & \\
\hline & $x$ & N.g & N.g & N.g & N.g & N.g & N.g & \\
\hline هـ و ز & $x$ & N.g & N.g & N.g & N.g & N.g & N.g & \\
\hline & $x$ & N.g & N.g & N.g & N.g & N.g & N.g & \\
\hline & $x$ & N.g & N.g & N.g & N.g & N.g & N.g & \\
\hline & $x$ & N.g & N.g & N.g & N.g & N.g & N.g & \\
\hline
\end{tabular}

ويلاحظ ان اسنَخدام درجة الحرارة

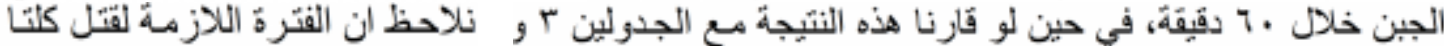

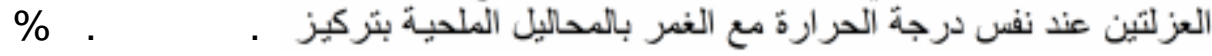




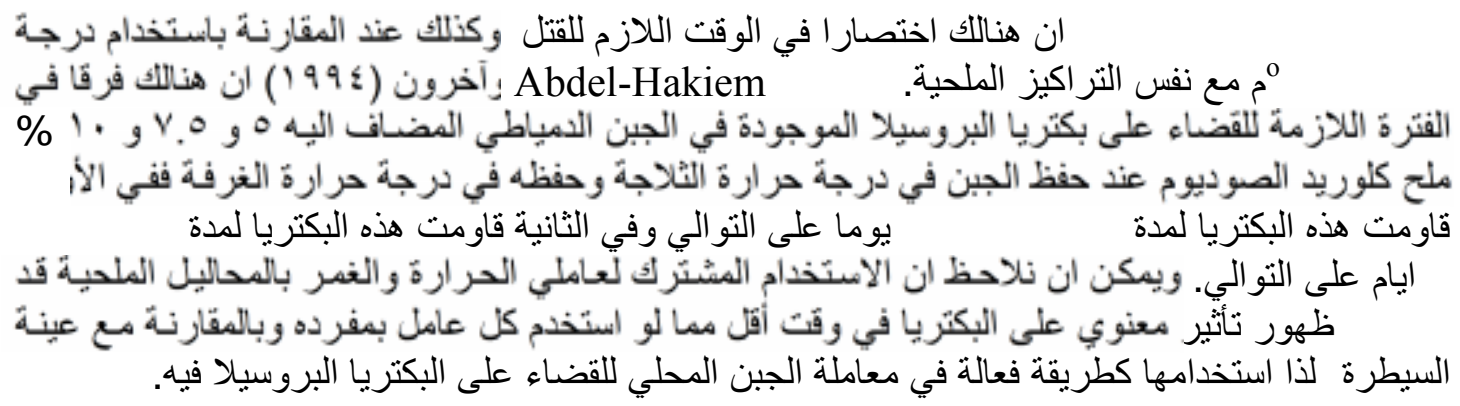

\title{
EFFECT OF SODIUM CHLORID AND TEMPERATURE ON ISOLATED Brucella melitensis FROM HUMAN BLOOD AND SHEEP MILK
}

Younis Ali Younis

Tariq Zaid Ibraheem

Food Sci. and Biotech. Dept., College of Agric. And Foresty, Mosul Univ., Iraq.

\begin{abstract}
Brucella melitensis was isolated from human which is the final host for this bacteria and from sheep milk, the main sourse of infection. Two isolates were obtained; isolate (1) from 23 blood speciemens taken from patients showed clinical findings of malta fever which their serum gave high agglutination titer in Rose Bengal Test, isolate (2) was isolated from 37 milk sample collected from (Mahweer, Manarat Shabbak and Bartillah) from sheep which suffered from abortion and their serum gave positive results with Rose Bengal Test. After the diagnostic biochemical tests, we found that these two isolates belonged to the species B. melitensis. Both isolates were cured in soft white cheese when flooded in a salt solution at concentrations 5 and $7.5 \%$ and stored at $4{ }^{\circ} \mathrm{C}$. No growth of isolate (1) was observed after 13, 11 days respectively, also no growth of isolate (2) was observed after 17,13 days when flooded with a salt solution at concentrations $5,7.5 \%$, respectively, but there was no effect of the concentration $2.5 \%$ on the resistant of both isolates in cheese. Both isolates were cured from soft white cheese when exposed to $50{ }^{\circ} \mathrm{C}$ for 60 minutes and $60{ }^{\circ} \mathrm{C}$ for 30 minutes. The study showed that the combination use of flooding with salt solution and high temperature in results treatment of both isolates in cheese has given an inhibitory effect on both isolates resistance in a short time when compared with the use of each factor alone. The concentration $2.5 \%$ showed an effect on both isolates in cheese when combined with the temperature factor. The use of $50{ }^{\circ} \mathrm{C}$ with flooding with salt concentrations $2.5,5,7.5 \%$ led to cure both isolates after $20,20,10$ minutes, respectively, and when $60{ }^{\circ} \mathrm{C}$ was used with the same concentrations above also led to cure both isolates at $10,10,5$ minutes respectively.
\end{abstract}

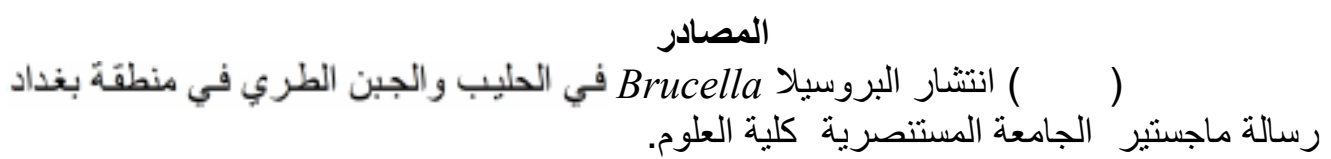

Abdel-Hakiem, E. H. ; M. E. Hamdy and M. A. Shelaih (1994). Viability of Brucella melitensis in damietta cheese, Assiut Vet. Med. J., 31 : 158-165.

Abtahi, H. ; A. H. Salmanian ; S. Rafati ; G. B. Nejad and Z. M. Hassan (2004). High level expression of recombinant ribosomal protein (L7 / L12) from 


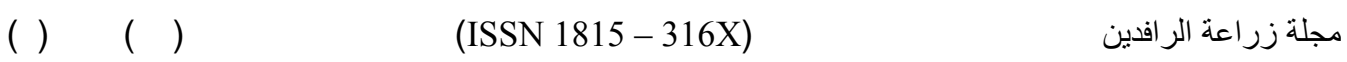

Brucella abortus and its reaction with infected human sera, Iran. Biomed. J., $8: 13-18$.

Alton, G. G. ; L. M. Jones ; R. D. Angus and J. M. Verger (1988).Techniques for the brucellosis laboratory, INRA. Paris.

Ayres, J. C. ; J. O. Mundt and W. E. Sandine (1980). Microbiology of foods, W. H. Freeman and Company.

Benenson, A. S. (1995). Control of communicable diseases manual, $16^{\text {th }}$ ed., American Public Health Association, Washington.

Billard ,E. ; J. Dornand and A. Gross (2007).Brucella suis Prevents Human Dendritic Cell Maturation and Antigen Presentation thrgh Regulation of Tumor Necrosis factor Alpha Secretion, Infect. Immun., 75 : 4980 - 4989.

Bricker, B.; D. R. Ewalt ; A. MacMillan; G. Foster and S. Brews (2000). Molecular characterization of Brucella strains isolated from marine mamals, J. Clin. Microbiol. , 38 : 1258-1262.

Center for food security and public health (2003). Brucellosis, 1-4, http:// www. Pnas.org/cgi/doi.

Collee, J. G. ; A. G. Fraser ; B. P. Marmion and A. Simmons (1996).Practical medical microbiology, $14^{\text {th }}$ ed. , Churchill Livingstone Inc., New York.

Corbel, M. J. (1997). brucellosis : An overview, Emerg. Infect. Dis., 3 : 213 - 221.

Fernandez - Lago, L. ; F. J. Vallejo ; I. Trujillano and N. Vizcaino (2001). Flourescent whole-cell hybridization with 16S rRNA - targated oligonucleotide probes to identify Brucella spp. by flow cytometry, J. Clinical Microbiology, 38 : 2768-2771.

Gandara, B. ; A. L. Merino ; M. A. Rogel and E. M. Romero (2001). Limited genitic diversity of Brucella spp., J. Clin. Microbiol. , 39 : 235 - 240.

John, G. H. ; N. R. Kreig ; H. A. Sneath ; J. T. Staley and S. J. Williams (1994). Bergey's manual of determinative bacteriology, $9^{\text {th }}$ ed. , Williams and Wilkins, U. S. A.

Johnson, E. A. ; J. H. Nelson and M. E. Johnson (1991). To pasteurize or not to pasteuriz, UW Dairy Pipeline, $3: 1-5$.

Kosikowiski, F. (1975). Cheese and fermented milk foods, Edfwards Brother, Inc. Ann. Arbor, Michigan.

Madigan, M. T. ; J. M. Martinko and J. Parker (2003). Biology of microorganism, $10^{\text {th }}$ ed. Pearson education, Inc.

Nicoletti, P. (1980). The epidemiology of bovine brucellosis, Adv. Vet. Sci. Comp. Med. 24 : 69-98.

Nimri, L. F. (2003). Diagnosis of recent and relapsed cases of human brucellosis by PCR assay, Bio Med Central, http://www.biomedcentral.com/147-2334/3/5.

Shehata, A. ; S. M. Adib and A. A. Al-Anzi (2001). Risk factor and clinical presentation of brucellosis in Al-Jahra hospital (1997 - 1999), Kuwait Med. J. , $33: 44-47$.

Soker, M. ; C. Devecioglu ; A. Yaramis ; S. Ipek ; M. N. Ozbek and H. Tuzuu (2001). Microangipathic hemolytic anemia, thrombocytopenia and acute renal failure associated with acute brucellosis, International Pediatrics, 16 : $105-108$. 